\title{
Targeting Leishmania: Revisiting Leishmania Iron Transporter 1 (LIT1)
}

\author{
Erin N White ${ }^{1}$, Evandrew Washington ${ }^{2}$ and Lawrence 0 Flowers ${ }^{3 *}$ \\ ${ }^{1}$ Associate Professor of Biology, Department of Biological and Forensic Sciences, Fayetteville State University, United States \\ ${ }^{2}$ Laboratory Manager, Department of Biological and Forensic Sciences, Fayetteville State University, United States \\ ${ }^{3}$ Associate Professor of Biology, Biology Department, Livingstone College, United States
}

*Corresponding author: Lawrence O Flowers, Biology Department, Livingstone College 209 Duncan Science Building,

701 West Monroe Street, Salisbury, NC 28144, United States

ARTICLE INFO
Received: 㓞 February 12, 2020
Published: 业 February 25, 2021

Citation: Erin $\mathrm{N}$ White, Evandrew Washington, Lawrence O Flowers. Targeting Leishmania: Revisiting Leishmania Iron Transporter 1 (LIT1). Biomed J Sci \& Tech Res 34(2)-2021. BJSTR. MS.ID.005517.

Keywords: Leishmania; LIT1; Case Fatality Rate; Iron Metabolism

\begin{abstract}
Leishmania is an obligate intracellular protozoan pathogen and a potential agent of bioterrorism. Leishmania is one of many parasitic protozoa responsible for high death rates in many parts of the world. Leishmania disease outcomes range from mild to severe and account for significant medical problems, especially in developing nations. Leishmania exists in two distinct morphological forms: promastigotes and amastigotes. Promastigotes resemble elongated flagellated cells that infect humans via sandfly vectors. Amastigotes are non-flagellated spherical cells that replicate asexually within human macrophages. Promastigotes differentiate into amastigotes inside macrophages and are transferred back to sandflies during subsequent blood meals. In the current commentary, we examine Leishmania iron transporter 1 (LIT1), a novel Leishmania membrane protein that plays a role in iron transport and parasite survival. Future investigations will explore the functional role of LIT1 in iron metabolism and other biologic processes and may lead to effective therapeutic strategies for treating Leishmaniasis and other parasitic infections.
\end{abstract}

Abbreviations: LIT1: Leishmania Iron Transporter 1; CFR: Case Fatality Rate; AIDS: Acquired Immune Deficiency Syndrome; ATP: Adenosine Triphosphate

\section{Introduction}

Intracellular pathogens are microorganisms that traverse the plasma membrane of a particular host cell. Once inside the host cell, intracellular pathogens successfully grow and reproduce within the host cell. The inhabitation of the pathogen typically promotes abnormal cell function and even destruction of the host cell. Intracellular pathogens are the etiological agents for various diseases, including African Sleeping-Sickness, Chagas' disease, and Leishmaniasis $[1,2]$. The threat of these types of diseases is apparent given the number of drug effectiveness studies performed in 2020 [3]. Understanding the cellular mechanisms that promote the intracellular survival of medically relevant pathogens is of great importance to life scientists. Members of the genus Leishmania are transmitted to humans by the bite of an infected sandfly. During a blood meal, the sandfly releases metacyclic promastigotes into the mammalian host. Promastigotes then invade mononuclear phagocytes and transform into amastigotes, the amplification stage of the parasite. Following repeated rounds of replication by binary fission, amastigotes are released from their cellular environment enabling the parasite to infect other host cells [4]. Sandflies ingest Leishmania amastigotes from infected mammalian hosts during subsequent blood meals. Within the sandfly's midgut tissue, amastigotes metamorphose into promastigotes and are eventually transmitted to another susceptible mammalian host during future blood meals.

Leishmania infections typically lead to three common types of clinical conditions in humans depending on the location of the infection, species, and the immunological state of the individual: cutaneous leishmaniasis, mucocutaneous leishmaniasis, and visceral leishmaniasis [5]. Leishmaniasis is one of the world's most devastating infectious diseases and, compared to other 
protozoan diseases, has been shown to have higher case fatality rates (Figure 1). The case fatality rate (CFR) is an epidemiological parameter that estimates the number of deaths from confirmed positive disease cases. CFRs proffer information regarding the public health threat and are used in classification schemes [6]. Leishmaniasis is also considered an opportunistic disease and is a significant cause of death in persons suffering from acquired immune deficiency syndrome (AIDS). With recent military efforts worldwide, the spread of leishmaniasis to U.S. military personnel has become a legitimate concern. At present, there are no effective human vaccines for the treatment of leishmaniasis. Leishmaniahost cell interactions are well coordinated molecular gambits involving ligands on the surface of the protozoan parasite and cell surface host proteins $[7,8]$. Transition into vertebrate cellular environments not only provides a strategy for parasite amplification and avoidance of immune detection, but intracellular invasion also allows Leishmania to be transported to secondary and tertiary sites within the mammalian anatomic framework.

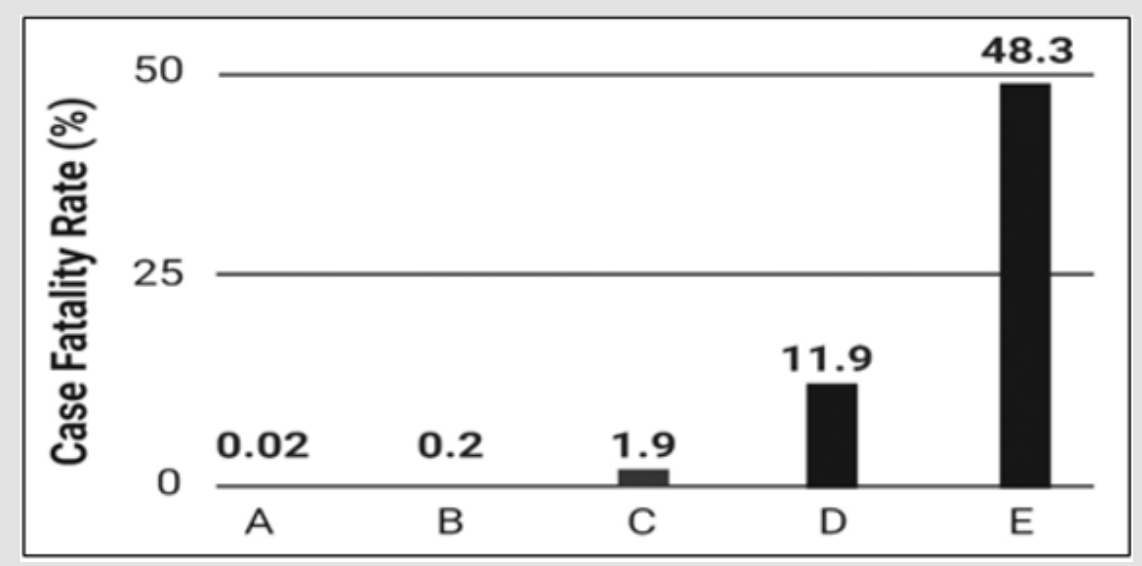

Figure 1: Case fatality rates (CFR) for protozoan parasites. The figure shows low to high case fatality rates expressed as percentages for
(A) schistosomiasis,
(B) malaria,
(C) cutaneous leishmaniasis,
(D) visceral leishmaniasis, and
(E) trypanosomiasis.

Immune responses to Leishmania involve cytokine activation of signaling pathways that promote apoptosis of phagocytes, nitric oxide production, inflammation, and proliferation of Type $1 \mathrm{~T}$ helper (Th1) cells. Many studies have demonstrated that Leishmania parasites block or activate essential signal transduction pathways that mediate antileishmanial processes and thereby evade host cell intracellular parasite suppression mechanisms [9,10]. Specifically, earlier studies have demonstrated that Leishmania can block the JAK-STAT signaling pathway and other signaling pathways to enhance infection, survival, and proliferation in host macrophages [11-13]. Several Leishmania species have been sequenced, which have allowed molecular parasitologists opportunities to investigate critical genetic mechanisms and protein structure.

\section{Leishmania Iron Transporter 1 (LIT1)}

Iron metabolism is paramount for intracellular parasites, including Leishmania, Trypanosoma, and Schistosoma [14]. Iron metabolism involves the transportation of useable forms of iron to specific cells and biological compartments. Iron utilization mediates homeostasis, promotes critical physiologic processes, and facilitates parasite replication. Iron also mediates the precise functioning of essential metabolic processes, including adenosine triphosphate (ATP) biosynthesis. Despite the enormous indispensability of iron, Leishmania cannot produce iron in vivo [15]. Additionally, the kinetoplastid parasites cannot store iron for later use. Macrophages engage in strategies to limit iron availability during intracellular habitation to starve infectious agents, thereby limiting growth and increasing innate immunity mediated by signal transduction pathways [16]. For Leishmania species, iron transport is further complicated because the protozoan must compete for iron with the host macrophage. The intraspecific competition for iron between the protozoan parasite and human cells is intriguing and the subject of several informative review articles $[17,18]$. Studies show that iron uptake and storage are essential for amastigote amplification within the phagocytic vacuole.

LIT1 is a Leishmania-specific membrane protein implicated in the recruitment of iron ions $\left(\mathrm{Fe}^{2+}\right)$ inside the macrophage [19]. 
LIT1 promotes efficient uptake of iron, which is essential for Leishmania survival and replication inside the macrophage's harsh intracellular compartment $[20,21]$. Putative LIT1 functionality was elucidated when using sequence homology; it was determined that the IRT1 (iron-regulated transporter 1) gene in Arabidopsis thaliana was similar [20]. Accordingly, LIT1 and IRT1 are members of the ZIP protein family that consist of eukaryotic metal transport transmembrane polypeptides with extensive extracellular and cytoplasmic peptide components [22]. Seminal investigations involving LIT1 mutational analysis provided clues as to the functional aspects of LIT1. Using Leishmania amazonensis, researchers showed that LIT1 gene deletions were sufficient to decrease amastigote proliferation. Reduction of amastigotes also reduces virulence as the outward appearance of LIT1 deletion mice showed less cutaneous wounds than mice infected with wild-type parasites [20]. It is unknown if LIT1 mutations or LIT1 gene deletions impact amastigote translocation to the arthropod vector or morphologic transformation in the sandfly. Currently, there are no studies that examine the use of chemicals or molecular therapeutics to target LIT1 precisely; however, studies have explored using quercetin to inhibit iron metabolism. In one such study, quercetin was shown to suppress amastigote division by altering a key enzyme in iron metabolism, ribonucleotide reductase [23].

\section{Conclusion}

Leishmania species are pathogenic protozoans that have evolved as successful intracellular parasites that preferentially target mammalian macrophages and other immune cellular effectors. Leishmania continues to infect and kill humans every year in high numbers. Vector control and drug regimens form the basis of leishmaniasis reduction methods. Once inside the host cell, intracellular pathogens grow and reproduce. The inhabitation of the pathogen typically promotes abnormal cell function and even destruction of the host cell. Each form of Leishmania is morphologically distinct and highly adapted for survival in the host. LIT1 proteins of Leishmania species are vital proteins that regulate iron metabolism in these intramacrophage parasites. Comparing the amino acid sequences of LIT1 proteins across different Leishmania species will expand our understanding of the LIT1 protein structure. Analysis of the amino acid sequences will also improve our comprehension of the function of LIT1 and help biologists identify similar proteins in the trypanosomatid family and other eukaryotic species. No drugs or molecular compounds have been shown to bind directly to LIT1 and reduce its effectiveness as an iron transporter. However, it may be possible to use amino acid sequence data and protein informatics software to search for potential LIT1 binding partners to treat leishmaniasis.

In this commentary, the authors focused on LIT1 as a potential therapeutic target. In addition to targeting LIT1, other complementary proteins such as the Leishmania iron regulator 1 (LIR1), a cytoplasmic-membrane protein found in promastigotes and amastigotes that prevents iron toxicity [24] and ferric reductase (LFR1), an enzyme that transfers an electron to iron (e.g., $\mathrm{Fe}^{3+}$ to $\mathrm{Fe}^{2+}$ ) are also latent targets [15]. It is also quite possible to employ an mRNA vaccine strategy similar to the currently used approach to defeat the COVID-19 pandemic [25] and generate an mRNA vaccine that directs the human body to produce antibodies to identify and eliminate trypanosomatid protein signatures crucial in iron metabolism. Targeting LIT1 and other proteins involved in iron homeostasis may be beneficial to disrupting essential Leishmania propagation processes.

\section{Acknowledgement}

This work was supported by a Livingstone College grant funded by the National Science Foundation (HRD-1533536).

\section{References}

1. Kaufer A, Ellis J, Stark D, Barratt J (2017) The evolution of trypanosomatid taxonomy. Parasites \& Vectors 10(1): 287.

2. Walker D, Oghumu S, Gupta G, Mc Gwire B, Drew M, et al. (2014) Mechanisms of cellular invasion by intracellular parasites. Cellular and Molecular Life Sciences 71(7): 1245-1263.

3. Peniche A, Osorio E, Melby P, Travi B (2020) Efficacy of histamine H1 receptor antagonists azelastine and fexofenadine against cutaneous Leishmania major infection. Public Library of Science Neglected Tropical Diseases 14(8): 1-17.

4. Chang K, Reed S, Mc Gwire B, Soong L (2003) Leishmania model for microbial virulence: The relevance of parasite multiplication and pathoantigenicity. Acta Tropica 85(3): 375-390.

5. Mansueto P, Vitale G, Di Lorenzo G, Rini GB, Mansueto S, et al. (2007) Immunopathology of leishmaniasis: An update. International Journal of Immunopathology and Pharmacology 20(3): 435-445.

6. Kanyina E (2020) Characterization of visceral leishmaniasis outbreak, Marsabit County, Kenya, 2014. BMC Public Health 20(1): 1-8.

7. Almeida MC, Vilhena V, Barral A, Barral Netto M (2003) Leishmanial infection: Analysis of its first steps. A review. Memorias do Instituto Oswaldo Cruz 98(7): 861-870.

8. Conceição Silva F, Morgado FN (2019) Leishmania spp-host interaction: There is always an onset, but is there an end? Frontiers in Cellular and Infection Microbiology 9: 1-14.

9. Olivier M, Gregory D, Forget G (2005) Subversion mechanisms by which Leishmania parasites can escape the host immune response: A signaling point of view. Clinical Microbiology Reviews 18(2): 293-305.

10. Forget G, Gregory DJ, Whitcombe L A, Olivier M (2006) Role of host protein tyrosine phosphatase SHP-1 in Leishmania donovani-induced inhibition of nitric oxide production. Infection and Immunity $74(11)$ : 6272-6279.

11. Forget G, Gregory DJ, Olivier M (2005) Proteasome-mediated degradation of STAT1alpha following infection of macrophages with Leishmania donovani. Journal of Biological Chemistry 280(34): 30542-30549.

12. Denkers E, Butcher B (2005) Sabotage and exploitation in macrophages parasitized by intracellular protozoans. Trends in Parasitology 21(1): 35-41.

13. Gazzinelli RT, Denkers EY (2006) Protozoan encounters with toll-like receptor signalling pathways: Implications for host parasitism. Nature Reviews Immunology 6(12): 895-906.

14. Glanfield A, Mc Manus DP, Anderson GJ, Jones MK (2007) Pumping iron: A potential target for novel therapeutics against schistosomes. Trends in Parasitology 23(12): 583-588. 
15. Laranjeira Silva M, Hamza I, Pérez Victoria J (2020) Iron and heme metabolism at the Leishmania-host interface. Trends in Parasitology 36(3): 279-289.

16. Nairz M, Haschka D, Demetz E, Weiss G (2014) Iron at the interface of immunity and infection. Frontiers in Pharmacology 5: 1-10.

17. Zaidi A, Singh K, Ali V (2017) Leishmania and its quest for iron: An update and overview. Molecular and Biochemical Parasitology 211: 1525.

18. Leon Sicairos N, Reyes Cortes R, Guadrón Llanos AM, Madueña Molina J, Leon Sicairos C, et al. (2015) Strategies of intracellular pathogens for obtaining iron from the environment. BioMed Research International 2015: 476534 .

19. Jacques I, Andrews NW, Huynh C (2010) Functional characterization of LIT1, the Leishmania amazonensis ferrous iron transporter. Molecular and Biochemical Parasitology 170(1): 28-36.

20. Huynh C, Sacks D, Andrews N (2006) A Leishmania amazonensis ZIP family iron transporter is essential for parasite replication within macrophage phagolysosomes. Journal of Experimental Medicine 203(10): 2363-2375

\section{ISSN: 2574-1241}

DOI: $10.26717 /$ BJSTR.2021.34.005517

Lawrence 0 Flowers. Biomed J Sci \& Tech Res

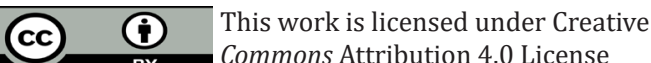

Submission Link: https://biomedres.us/submit-manuscript.php
21. Flannery AR, Renberg RL, Andrews NW (2013) Pathways of iron acquisition and utilization in Leishmania. Current Opinion in Microbiology 16(6): 716-721.

22. Guerinot ML (2000) The ZIP family of metal transporters. Biochimica et Biophysica Acta 1465: 190-198.

23. Sen G, Mukhopadhyay S, Ray M, Biswas T (2008) Quercetin interferes with iron metabolism in Leishmania donovani and targets ribonucleotide reductase to exert leishmanicidal activity. Journal of Antimicrobial Chemotherapy 61(5): 1066-1075.

24. Laranjeira Silva MF, Wang W, Samuel TK, Maeda FY, Michailowsky V, et al. (2018) A MFS-like plasma membrane transporter required for Leishmania virulence protects the parasites from iron toxicity. Public Library of Science Pathogens 14(6): e1007140.

25. Ahammad I, Lira S (2020) Designing a novel mRNA vaccine against SARS-CoV-2:An immunoinformatics approach. International Journal of Biological Macromolecules 162: 820-837.

$\begin{array}{ll}\text { BIOMEDICAL } & \text { Assets of Publishing with us } \\ \text { RESEARCHES } & \text { - Global archiving of articles } \\ \text { - Immediate, unrestricted online access } \\ \text { - Rigorous Peer Review Process }\end{array}$

